\title{
La profilaxis pre-exposición reduciría la incidencia de infecciones por HIV en personas con conductas de alto riesgo
}

\author{
Pre-exposure prophylaxis would reduce the incidence of HIV infections in people with high-risk behaviors
}

\section{Objetivos}

Demostrar la eficacia y la seguridad de la profilaxis pre-exposición (PrEP) según la actividad sexual ("a demanda") con la combinación de los antirretrovirales tenofovir/emtricitabina (siglas técnicas: TDF/FTC) en hombres y trans femeninas que tienen sexo anal no protegido.

\section{Diseño, lugar y métodos}

Ensayo clínico doble ciego, aleatorizado, de PrEP para la infección por el virus de la inmunodeficiencia humana (en inglés HIV-1) en hombres y mujeres transexuales de Francia y Canadá seronegativos para el HIV con alto riesgo de infección por tener sexo anal no protegido. La aleatorización se realizó en bloques de cuatro, estratificados por país. En el enrolamiento, los participantes elegibles sin infección por HIV fueron asignados en una proporción de 1:1 a recibir TDF-FTC o placebo.

\section{Intervención}

A los pacientes con alto riesgo de infección -antecedente de relaciones sexuales anales sin protección con al menos dos personas diferentes en los últimos seis meses-, se les asignó a recibir TDF/ FTC o placebo en cada exposición sexual con el siguiente esquema: dos comprimidos juntos entre 24 y 2 horas antes del contacto sexual, un comprimido 24 horas después de los primeros, y un cuarto comprimido 24 horas después del tercero. En el caso de contactos sexuales consecutivos, deberían seguir tomando un comprimido al día hasta el último contacto y luego tomar dos comprimidos más, separados por 24 horas. Asimismo, si entre un contacto sexual y otro
Molina J y col. N Engl J Med. 2015;373(23):2237-46. transcurría menos de una semana, sólo se tomaría un comprimido pre-exposición, y luego los dos post-exposición. Todos los participantes recibieron formación sobre las medidas de prevención, se les proporcionó preservativos, realizaron pruebas detección de infecciones de transmisión sexual y se les indicó profilaxis post-exposición ante un contacto sexual con un individuo con infección por HIV.

\section{Medición de resultados principales}

El análisis primario se hizo por intención de tratar, excluyendo los datos de los pacientes a quienes se les detectó la infección por HIV antes de recibir la primera dosis de la medicación del estudio, a los que fueron perdidos en el seguimiento y a los que retiraron el consentimiento entre la aleatorización y el enrolamiento y no recibieron la medicación del estudio. Se pautaron visitas de seguimiento a las cuatro y ocho semanas, en las que se realizó testeo de HIV mediante ELISA de cuarta generación (detecta antígeno p24 de infección temprana)

\section{Resultados}

El comité externo de seguridad detuvo precozmente la rama de placebo cuando se llevaban aleatorizados 414 sujetos, de los cuales 400 llegaron a recibir la intervención, con una mediana de seguimiento de 9,3 meses (rango intercuartílico 4,9 a 20,6 meses). Dieciséis participantes se infectaron por el VIH durante el seguimiento: dos en el grupo de tratamiento (incidencia 0,91 por 100 personasaño) y 14 en el grupo placebo (incidencia 6,6 por 100 personas-año), lo que supone una reducción relativa del riesgo de infección del $86 \%$ (IC95\%, 40 a 98\%; p=0,002). Ver Tabla 1.

Tabla 1. Eficacia de la profilaxis pre-exposición de HIV en población de alto riesgo.

\begin{tabular}{|c|c|c|c|}
\hline & \multicolumn{2}{|c|}{ Nuevos casos (densidad de incidencia en personas-año) } & \multirow[b]{2}{*}{$\begin{array}{c}\text { Reducción en la } \\
\text { tasa de infección (IC 95\%) }\end{array}$} \\
\hline & Profilaxis pre-exposición & Placebo & \\
\hline $\begin{array}{l}\text { Hombres que tienen sexo con hombres y trans } \\
\text { femeninas con conductas de alto riesgo }\end{array}$ & $\begin{array}{c}2 / 199 \\
(0,91 / 100)\end{array}$ & $\begin{array}{c}14 / 201 \\
(6,6 / 100)\end{array}$ & $\begin{array}{c}86 \% \\
(40 \text { a } 98 \%)\end{array}$ \\
\hline
\end{tabular}

Los dos infectados en el grupo PrEP tuvieron una adherencia, valorada por la medicación devuelta, prácticamente nula. Por término medio, cada participante tomó 15 comprimidos al mes (rango intercuartílico 11 a 21), aunque se apreció una alta variabilidad inter e intraindividual a lo largo del seguimiento.

\section{Conclusiones}

El uso de PrEP (TDF-FTC) antes y después de las relaciones

sexuales provee protección contra la infección por HIV-1 en hombres que tienen sexo con hombres y trans femeninas con conductas de alto riesgo. El tratamiento se asoció con el aumento de los efectos adversos renales y gastrointestinales.

Fuente de financiamiento: ANRS, Red canadiense de ensayos sobre HIV, Fondo Pierre Bergé de Donación para la Prevención, y la Fundación Bill y Melinda Gates. Gilead Sciences donó TDF-FTC y placebo usado en el estudio y la financiación para el análisis farmacocinético.

\section{Comentario}

Éste y otros ensayos clínicos y estudios observacionales confirman la eficacia y la efectividad de la PrEP basada en TDF/FTC en personas con elevado riesgo ${ }^{1}$. La eficacia depende de la adherencia, habiéndose establecido un umbral mínimo de cuatro comprimidos por semana para mantener una eficacia elevada ${ }^{2}$. Es posible también utilizar pautas intermitentes, asociadas al momento del contacto de riesgo, aunque probablemente sólo serán una alternativa en personas con una baja frecuencia de exposiciones de riesgo ${ }^{3}$.

\section{Conclusiones de la comentadora}

En poblaciones de alto riesgo podemos recomendar la profilaxis pre exposición teniendo en cuenta que puede asociarse a un aumento de la toxicidad. Por otro lado, la PrEP debería prescribirse dentro de un plan de prevención global que incluya consejo asistido y formación. Dado los costos de esta estrategia, son candidatos para recibir PrEP las personas pertenecientes a poblaciones con una incidencia de infección por HIV mayor a dos casos por cada 100 personas-año. El inicio de la PrEP debe conllevar una evaluación individual del riesgo de infección por el HIV.

Verónica Cisneros [ FUNCEI, Fundación Centro de Estudios Infectológicos. verocisneros@gmail.com ]

Cisneros V. La profilaxis pre-exposición reduciría la incidencia de infecciones por HIV en personas con conductas de alto riesgo. Evid Act Pract Ambul. 2017; 20(4):95. Comentado de: Molina J, y col. On-Demand Preexposure Prophylaxis in Men at High Risk for HIV-1 Infection. N Engl J Med. 2015;373(23):2237-46. PMID: 26624850.

Referencias

1. Mccormack $S$ y col. Pre-exposure prophylaxis to prevent the acquisition of HIV-1 infection (PROUD): eff ectiveness results from the pilot phase of a pragmatic open-label randomised trial. Lancet. 2015;6736(15): 1-8.

2. GESIDA, Grupo de Estudio de Sida de la SEIMC. Recomendaciones sobre Profilaxis Pre-Exposición en adultos para la Prevención de la Infección por VIH en España [Internet]. 2016 [cited 2018 Jan 25]. Disponible en URL: http://www.cesida.org/wp-content/uploads/2013/09/gesida-guiasclinicas-2016-profilaxis_pre-exposicionVIH.pdf 3. Amico KR y col. Experiences with HPTN 067/ADAPT Study-Provided Open-Label PrEP Among Women in Cape Town: Facilitators and Barriers Within a Mutuality Framework. AIDS Behav. 2017;21(5):1361-75. PMID: https://www.ncbi.nlm.nih.gov/pubmed/27317411. 